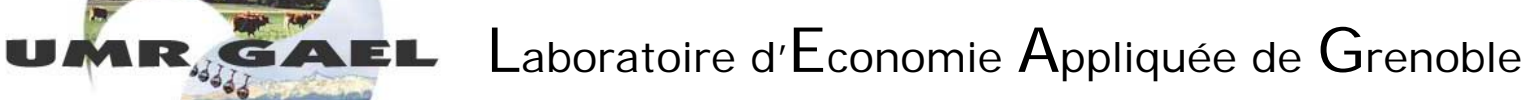
alite

LES PRODUITS DE TERROIR : VECTEURS DE VALORISATION DES AMENITES?

KREZIAK Dominique ; LACROIX Anne ; LENGLET François

- October 2010 -

JEL CODES : C93 ; D12 ; Q26 ; Q51

Working Paper GAEL ; 2010-06 


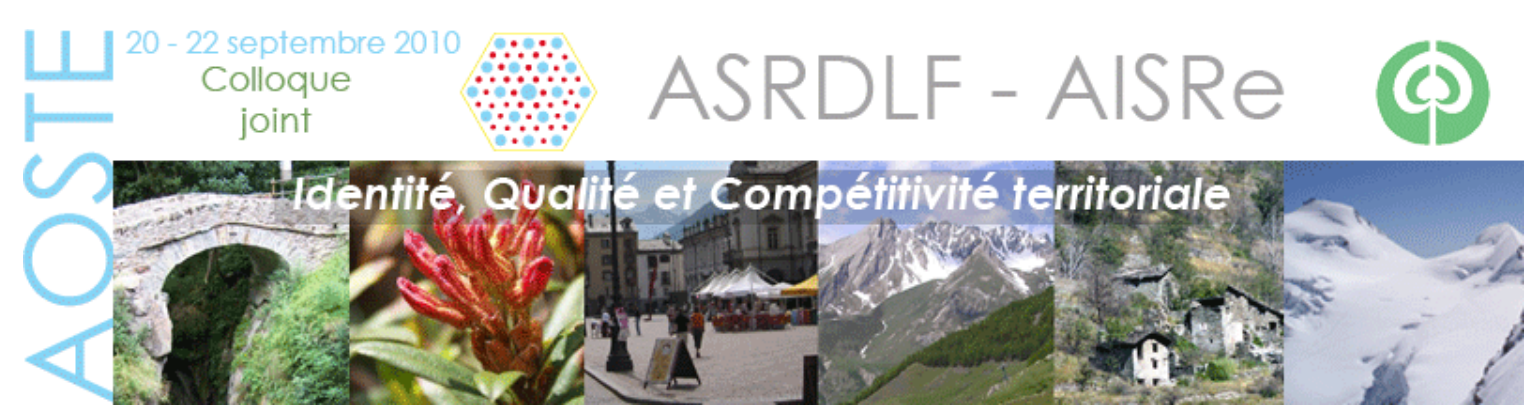

\title{
LES PRODUITS DE TERROIR : VECTEURS DE VALORISATION DES AMENITES?
}

\author{
KREZIAK Dominique \\ IREGE, Université de Savoie \\ Chambéry \\ dominique.kreziak@univ-savoie.fr \\ LACROIX Anne \\ UMR GAEL INRA/Université Pierre Mendès \\ France \\ Grenoble \\ lacroix@grenoble.inra.fr \\ LENGLET François \\ IREGE, Université de Savoie \\ Annecy \\ francois.lenglet@univ-savoie.fr
}

\section{Résumé :}

Ce travail constitue une première approche des aménités et de leur valorisation potentielle via les produits alimentaires. Plus exactement, il s'agit de savoir si la valeur accordée à un produit issu d'une région bénéficiant d'un environnement naturel typé est plus importante que celle accordée à un produit issu d'une région dont l'environnement est moins attractif. Pour répondre à cette question, nous recourrons à une analyse en laboratoire qui compare deux fromages issus de deux territoires des Alpes françaises. Les résultats montrent notamment que les aménités d'un territoire, même si elles participent de la valeur affective d'un produit, ne peuvent être valorisées économiquement que s'il y a congruence entre le territoire et le produit.

\section{Mots clés :}

Aménités, Consentement à payer, évaluation hédonique, produit de terroir

Classification JEL :

C93, D12, Q26, Q51 


\section{LES PRODUITS DE TERROIR : VECTEURS DE VALORISATION DES AMENITES ?}

\section{INTRODUCTION}

La recherche de stratégies politiques durables pour assurer l'avenir des zones rurales a révélé que les aménités naturelles et culturelles constituaient un domaine d'action important, complémentaire des politiques rurales traditionnelles orientées vers l'agriculture (OCDE, 1994, 1996, 1999). L'OCDE note alors que de nombreux potentiels naturels et culturels demeurent inexploités dans la plupart des zones rurales et que pour en tirer parti, il convient de trouver l'équilibre délicat entre l'offre et la demande. En somme, la valorisation des aménités est devenue l'enjeu des dynamiques des territoires ruraux et, plus largement, du développement territorial.

Concrètement, la contribution des aménités au développement local et régional se révèle au travers des comportements résidentiels, récréatifs et touristiques. En effet, les choix de résidence, de destination récréative ou touristique relèvent pour une grande part de préférences, fondées sur les aménités culturelles et paysagères qu'offrent les différents espaces (Poudyal et al., 2008 ; Chen et al., 2009 ; Morey, 1981). Plus encore, il a été montré à maintes reprises que les externalités environnementales impactent le prix des biens immobiliers. Ce résultat est acquis pour les externalités négatives telles que les pollutions (pour une revue, voir Boyle et Kiel, 2001), mais aussi pour les externalités positives comme les aménités. Dans ce cas, le prix du bien immobilier est supérieur dans un cadre avec aménités : présence d'arbres, d'espaces ouverts à proximité du bien ... que sans celles-ci (Cavailhès et al., 2009 ; Thorsnes, 2002). II a été montré aussi que les aménités peuvent être valorisées dans le prix d'un service touristique, par exemple la location d'un gîte (Le Goffe, 2000 ; Mollard et al., 2007) ou dans les dépenses effectuées pour des activités récréatives (Hanley et al., 2002 ; Crell et Loomis, 1992).

Mais à notre connaissance, aucune recherche ne s'est intéressée à l'impact des aménités sur les prix des produits alimentaires. Aussi l'objectif de ce papier vise à combler cette lacune en analysant si les produits de terroir constituent un vecteur de valorisation des aménités. Autrement dit, la question posée est la suivante : est-ce que la valeur accordée aux produits issus de régions bénéficiant d'un environnement naturel agréable est plus importante que celle accordée aux produits issus de régions dont l'environnement est moins attractif?

Tout au plus, certains travaux se sont attachés à analyser l'effet de l'image de la région de provenance des produits, sur les dépenses des consommateurs en vins régionaux (Skuras et Dimara, 2004) et plus largement en produits issus du territoire (Skuras et al., 2006). Les résultats mettent en évidence que l'image de la région influence le montant des dépenses. Mais si cette image se fonde sur les aménités ou la culture, alors son rôle n'est pas net sur la probabilité de faire de tels achats, ni sur le montant de ces achats. II est vraisemblable que la manière dont a été menée cette investigation (enquête déclarative en face-à-face, questions très larges sur les habitudes de consommation) a affecté les résultats.

Afin d'éviter ce biais, nous aurons recours à la méthode d'économie expérimentale qui consiste à créer un marché en univers contrôlé, afin d'observer de véritables comportements d'achat tout en contrôlant au mieux les variables décisionnelles qui pèsent sur ce comportement. La suite du papier est organisée comme suit: nous exposons la méthodologie en section 2 ; la section 3 présente les résultats et la section 4 les discute et conclut. 


\section{Methodologie}

\subsection{Considérations préalables}

Afin de rechercher si les produits de terroir constituent un vecteur de valorisation des aménités, nous nous appuyons sur une conception lancastérienne de la consommation. En effet, Lancaster, 1966 a introduit une importante modification à la théorie du consommateur en supposant que l'utilité procurée par la consommation d'un bien provenait en réalité de la consommation de ses caractéristiques ou attributs. Les caractéristiques d'un même bien étant identiques et fournies en quantité égale à tous les consommateurs, leurs préférences (en termes d'attributs) sont reflétées par leurs choix.

Nous considérons donc ici que le produit de terroir est constitué de différentes caractéristiques, dont celle relevant de l'agrément des aménités du territoire dont il est issu. Ceci nous conduit à considérer le produit de terroir comme un bien public impur (Kotchen, 2005) qui comprend à la fois des caractéristiques privées et une caractéristique environnementale publique.

Toutefois, cette caractéristique pourrait être confondue avec l'origine du produit qui, elle aussi, contribue à sa valeur. En effet, dans leur revue de la littérature en marketing, Verlegh et Steenkamp (1999) montrent qu'il existe une influence de l'origine géographique sur la valeur accordée à un produit ; ceci tient surtout au fait que la mention géographique agit comme un signal de qualité. Ce résultat est acquis tant pour une origine-pays que pour une origine plus délimitée (Stefani et al., 2006). Ces derniers auteurs mettent d'ailleurs en évidence que cet effet sur la valeur est d'autant plus important que le territoire d'origine est petit. Mais, au-delà du signal qualité, la zone géographique véhicule d'autres signaux plus affectifs, parmi lesquels figurent les aménités. Ainsi, Van Ittersum et al. (2003) distinguent deux dimensions principales du territoire : humaine (savoir-faire, identité...) et environnementale (nature, paysage ...).

L'objectif de notre recherche vise précisément à appréhender cette dimension environnementale indépendamment du signal qualité. Cependant, le concept d'aménités s'avère complexe et n'a guère fait l'objet d'investigations jusqu'alors (Mollard, 2010). II mêle des éléments objectifs de l'environnement à une perception subjective : un élément peut être agréable pour certaines personnes et ne procurer aucun bien-être pour d'autres. Aussi, convient-il que le protocole mis en place soit suffisamment précis et parvienne à mesurer la valeur de cet attribut, et plus généralement du produit, dans sa dimension affective et conative.

Le consommateur évalue le produit sur la base de ses attributs, lesquels correspondent à un ensemble de stimuli variés : sensoriels et épistémiques. La valeur d'un produit résulte alors des réponses affectives et conatives des consommateurs à ces stimuli. Une réponse affective est définie comme un sentiment, une impression; elle est prise en compte selon l'approche classique en marketing et sciences des aliments : attente hédonique si les stimuli sont uniquement épistémiques, évaluation hédonique globale si elle est basée sur l'ensemble des stimuli (Cohen et Areni, 1991). Par contre, les réponses conatives (élan poussant à l'action, impulsion active) s'inscrivent davantage dans une approche plus économique (intention d'achat); elles sont appréhendées via la mesure du consentement à payer (CAP) (voir LeGall-Ely, 2009 pour une revue).

\subsection{Protocole expérimental}

Deux produits ont été retenus : la Tome des Bauges et une tomme dite " de montagne » de l'Avant Pays Savoyard, de qualités sensorielles proches mais non-équivalentes. Un test d'étalonnage, effectué auprès de 54 consommateurs experts de fromage, a montré que $74,1 \%$ (test triangulaire 1 ) et $90,7 \%$ (test triangulaire 2) d'entre eux étaient capables de repérer les différences sensorielles entre ces deux tommes. 
Ces deux tommes sont produites en région de montagne, mais dans des territoires dotés de paysages différenciés. Dans la typologie de la DIREN Rhône-Alpes (2007), les paysages de l'Avant Pays Savoyard relèvent de paysages agraires ; ceux des Bauges, de paysages ruraux-patrimoniaux qui se distinguent des paysages agraires en raison de structures paysagères singulières qui leur confèrent une identité forte. Les aménités de chacun de ces territoires faisaient l'objet d'une évaluation à partir d'un jeu de (7X2) photos et via une mesure d'agrément (échelle de Likert de 1 à 7 ). Afin de ne pas influencer cette évaluation, la localisation des photos n'était pas indiquée et le protocole a permis de contrôler l'effet d'ordre. Les photos avaient trait à sept éléments des aménités visuelles : eau, paysage, végétation, pâturage, flore, architecture, village. Ces éléments avaient été déterminés au cours d'un travail exploratoire effectué en concertation avec des cogniticiens et des écologues : il s'agissait de choisir des photos qui soient pertinentes en termes de représentations cognitives, de représentations écologiques et de valeur économique. L'analyse des résultats via une analyse factorielle n'a pas révélé les sept dimensions attendues et nous a incités à retenir une approche unidimensionnelle des aménités : il n'existait pas d'élément saillant, mais une perception globale d'un système d'aménités environnementales.

Le protocole de l'expérience a procédé par information croissante, en se rapprochant le plus possible des conditions d'achat réelles et en allant de l'information la plus objective vers l'information la plus subjective:

- Phase 1 : présentation des processus de production de chacune des tommes (race et alimentation du cheptel laitier, mode et durée d'affinage ...) afin de capturer l'effet qualité ;

- Phase 2 : révélation des aménités environnementales de chacune des zones de production sur la base des photos qui avaient fait l'objet d'une appréciation au préalable ;

- Phase 3 : révélation de la zone de production et localisation sur une carte ;

- Phase 4 : dégustation informée de chacune des tommes.

En début d'expérience, un questionnaire conviait les participants à décrire leurs caractéristiques socio-démographiques et quelques unes de leurs habitudes alimentaires. En fin d'expérience, un autre questionnaire collectait leurs croyances à l'égard des prix de marché de chacune des tommes (prix de référence), leur fréquentation de chacun des territoires et les variables psychologiques.

\subsection{Mesure des construits}

A chacune des quatre phases, les participants devaient évaluer chacun des fromages (en termes d'attente ou de notation hédonique et de CAP exprimé en $€ / \mathrm{kg}$ ). L'ordre d'évaluation des tommes était contrôlé et après analyse des résultats, aucun effet d'ordre n'a été constaté.

L'évaluation hédonique est mesurée lors de la phase 4 avec une échelle discontinue numérique à 10 points pour " l'évaluation du plaisir momentané », décrite dans la norme NF V 09-015 consacrée aux techniques d'évaluation sensorielle. Elle fournit une note d'agrément à l'issue de la dégustation. Pour les attentes hédoniques, collectées à chaque étape, la mesure retenue consiste encore en une échelle de notation en 10 points destinée à enregistrer la réponse à la question : " A la vue de cette information, comment vous attendez-vous à aimer ce produit ? » (Levin et Gaeth, 1988 ; Spreng et al., 1996 ; Deliza, 1996 ; Schifferstein et al., 1999). Les attentes sont recueillies lors des phases 1 à 3.

Les CAP étaient révélés via une procédure de vente, appelée " enchère BDM » du nom de ses auteurs (Becker, DeGroot, Marschak, 1964), et recommandée pour ce type de produits (LeGall-Ely, 2009). Dans cette procédure, les acheteurs font une offre sous pli fermé. Une fois les offres collectées, un prix de vente effectif est tiré au sort dans un ensemble de prix possibles préalablement défini (dans notre cas, la loi de distribution était uniforme de 0 à 
$19,99 €)$. Les acheteurs ayant fait une offre de prix supérieure au prix tiré au sort achètent le produit au prix tiré au sort, les autres n'achètent pas. Ainsi, tous les participants, quel que soit le niveau auquel ils valorisent le bien, ont une probabilité non nulle d'acheter le bien à un prix inférieur à leur offre, ce qui constitue un mécanisme incitatif pertinent pour ce type de collecte. Dans notre expérience, les offres ont été collectées à chaque phase pour chacun des fromages. Afin de limiter les achats des sujets, à la fin de l'expérience, une seule des quatre phases et une des deux tommes ont été tirées au sort.

Par définition, la valeur d'un bien ou d'une caractéristique est perçue de manière très subjective. Pour rendre compte de cette hétérogénéité, on a en général recours aux descripteurs socio-démographiques (sexe, âge, profession...). Par leur nature physiologique, l'âge et le genre conduisent assez fréquemment à des différences perceptuelles (e.g. Cordelle et al., 2004). D'autres caractéristiques comme le revenu ou le niveau d'étude peuvent également être étudiées. De même, selon les théories de l'apprentissage, la familiarité par expositions répétées augmente les préférences, et la fréquence de consommation doit donc être contrôlée. Toutefois il paraît également utile d'explorer le pouvoir prédictif d'autres variables comme les caractéristiques psychologiques, qui représenteraient en la matière des critères de segmentation plus pertinents que les variables socio-démographiques (Abiven, 2004).

A ce titre, la tendance à rechercher la variété dans son alimentation est souvent perçue comme un facteur de motivation important (Sirieix, 1999 ; Lange, 2000). De même, l'attitude est depuis longtemps considérée comme l'un des principaux déterminants des comportements de consommation. Les motivations alimentaires correspondent à un " ensemble de considérations susceptibles d'être prises en compte par les individus pour choisir ce qu'ils vont manger "(Steptoe et al., 1995).Parmi celles-ci, on distingue en particulier :

- Les préoccupations de santé : le choix est motivé par le souci d'avoir une alimentation saine ;

- Les préoccupations éthiques : par exemple, la capacité de l'aliment à préserver l'environnement est considérée comme importante ;

- La nouveauté : le choix d'un produit nouveau ou inconnu est motivé par le besoin de sensation ou la curiosité.

Les mesures des construits psychologiques se réalisent classiquement au moyen d'échelles multi-items. Les choix d'opérationnalisation de ces construit conduisent à retenir des outils aux qualités psychométriques éprouvées et/ou adaptées à un contexte français (échelle de tendance à la recherche de variété en alimentaire Varseek : Van Trijp et Steenkamp, 1992, adaptation française : Liquet et Lenglet, 2002). Pour l'échelle d'attitude, la mesure est construite à partir de la liste des critères d'achat en alimentaire développée par Arts-Chiss et Guillon (2003).

Dans notre expérience, les qualités psychométriques des variables psychologiques sont bonnes voire très bonnes ( $\alpha$ de Cronbach : 0,604 à 0,720 pour les dimensions nouveauté, santé, et environnement de l'attitude ; 0,884 pour la tendance à la recherche de variété ; dimensionnalités conformes à la théorie. En conséquence, et pour chaque dimension, un score additif des items a été retenu comme mesure des construits.

\section{Resultats}

L'expérience a été réalisée, entre juin et juillet 2009, auprès de 179 sujets (cf. tableau 1), répartis en strates définies selon leur degré de fréquentation des Bauges : touristes, excursionnistes (habitants de la région qui vont au moins 1 fois par an dans le territoire) et neutres (habitants de la région qui n'y vont pas du tout ou moins d'une fois par an) et leur degré de fréquentation de l'Avant Pays Savoyard: excursionnistes, neutres. 
Tableau 1. Caractéristiques des participants à l'expérience.

\begin{tabular}{|c|c|c|}
\hline & Nombre & Fréquence (\%) \\
\hline $\begin{array}{c}\text { Sexe : homme } \\
\text { femme }\end{array}$ & $\begin{array}{l}82 \\
97\end{array}$ & $\begin{array}{l}45.81 \\
54.19\end{array}$ \\
\hline $\begin{aligned} \text { Age }: & 40 \text { ans et moins } \\
& \text { De } 41 \text { à } 60 \text { ans } \\
& \text { Plus de } 60 \text { ans }\end{aligned}$ & $\begin{array}{l}46 \\
87 \\
44\end{array}$ & $\begin{array}{l}25.99 \\
49.15 \\
24.86\end{array}$ \\
\hline $\begin{array}{l}\text { Niveau de diplôme : Pas le bac } \\
\text { Bac à bac+4 } \\
\text { Bac+5 et plus }\end{array}$ & $\begin{array}{l}60 \\
61 \\
55\end{array}$ & $\begin{array}{l}34.09 \\
34.66 \\
31.25\end{array}$ \\
\hline $\begin{array}{l}\text { Niveau de revenu par unité de consommation: } \\
\text { inférieur à } 1000 \frac{\mathrm{m}}{\mathrm{mois}} \\
\text { entre } 1000 \text { et } 2000 \% / \text { mois } \\
\text { supérieur à } 2000 \% / \text { mois }\end{array}$ & $\begin{array}{l}60 \\
66 \\
46\end{array}$ & $\begin{array}{l}34.88 \\
38.37 \\
26.74\end{array}$ \\
\hline $\begin{aligned} \text { Fréquentation des Bauges : excursionnistes } \\
\text { neutres } \\
\text { touristes }\end{aligned}$ & $\begin{array}{l}69 \\
52 \\
58\end{array}$ & $\begin{array}{l}38.55 \\
29.05 \\
32.40\end{array}$ \\
\hline $\begin{array}{l}\text { Fréquentation de l'Avant Pays Savoyard } \\
\text { excursionnistes } \\
\text { neutres }\end{array}$ & $\begin{array}{l}92 \\
84\end{array}$ & $\begin{array}{l}52.27 \\
47.73\end{array}$ \\
\hline $\begin{array}{l}\text { Consommation de fromage : A chaque repas } \\
\text { Une fois par jour } \\
\text { Moins d'une fois par jour }\end{array}$ & $\begin{array}{l}53 \\
81 \\
44\end{array}$ & $\begin{array}{l}29.78 \\
45.51 \\
24.72\end{array}$ \\
\hline $\begin{array}{l}\text { Fréquence de consommation de la tome des } \\
\text { Bauges } \\
\text { Au moins une fois par semaine } \\
\text { Moins souvent } \\
\text { Presque jamais }\end{array}$ & $\begin{array}{l}36 \\
87 \\
53\end{array}$ & $\begin{array}{l}20.45 \\
49.31 \\
30.11\end{array}$ \\
\hline
\end{tabular}

\subsection{Valeur relative des produits}

La tome des Bauges est systématiquement mieux évaluée que la tomme de montagne, que ce soit en termes de notation hédonique, de CAP, ou de prix de référence. Pour la tome des Bauges, les évaluations progressent avec l'information sur les aménités et la révélation de la région de production, mais le goût réduit tant la note hédonique que le CAP. Pour la tomme de montagne, l'impact de la description des aménités est moindre sur l'attente hédonique ; il est nul sur son CAP. La révélation de la zone de production fait progresser l'attente hédonique et le CAP ; le goût ne fait monter ni la note, ni le CAP (Tableau 2).

Tableau 2. Evaluation (moyenne +/- écart type) des deux produits au cours des différentes phases de l'expérience

\begin{tabular}{|c|c|c|c|c|}
\hline & $\begin{array}{c}\text { Phase 1: } \\
\text { processus } \\
\text { production }\end{array}$ & $\begin{array}{c}\text { Phase 2: } \\
\text { aménités }\end{array}$ & $\begin{array}{c}\text { Phase 3: } \\
\text { zone production }\end{array}$ & $\begin{array}{c}\text { Phase 4: } \\
\text { goût }\end{array}$ \\
\hline Tome des Bauges & & & & \\
\hline Attente ou note hédonique & & & & \\
\hline CA* & $7.04+/-1.78$ & $7.63+/-1.73$ & $7.90+/-1.69$ & $7.32+/-2.10$ \\
\hline Tomme de montagne & $10.27+/-5.52$ & $10.87+/-5.69$ & $11.18+/-5.69$ & $10.37+/-6.37$ \\
\hline Attente ou note hédonique (1) & $5.51+/-2.24$ & $5.75+/-2.12$ & $6.03+/-2.09$ & $6.03+/-2.30$ \\
\hline CAP (2) & $8.03+/-5.53$ & $8.04+/-5.50$ & $8.45+/-5.53$ & $8.32+/-5.44$ \\
\hline
\end{tabular}

(1) différences entre phase 1 et phase 2 , entre phase 2 et phase $3=* \star \star$

(2) différence entre phase 2 et phase $3=* \star \star$

${ }^{\star * \star} p<0.01$ d'après le test de Student de comparaison de moyennes sur échantillons appariés. 


\subsection{Déterminants de la valeur des produits}

Bien que les deux types de valeurs (valeur affective appréhendée via l'attente ou la note hédonique et valeur économique appréhendée via le CAP) soient très corrélées (coefficient de corrélation $=0.52$ pour chacune des tommes), leurs déterminants respectifs ne sont pas équivalents.

Une estimation économétrique (cf. liste variables en tableau 3) des notations hédoniques de chacun des fromages (cf. tableau 4) montre que le prix de référence, la note d'agrément attribuée aux aménités paysagères, le goût, l'information sur la région d'origine, le fait de fréquenter régulièrement le territoire (excursionniste) exercent une influence positive sur la valeur affective. A l'inverse, le fait de disposer d'un faible revenu exerce une influence négative. En outre, les touristes et les consommateurs en forte recherche de variété alimentaire pénalisent la valeur de la Tome des Bauges.

Tableau 3. Variables indépendantes utilisées dans l'estimation économétrique

\begin{tabular}{|c|c|}
\hline phamen & $\begin{array}{l}\text { Indicatrice : } 1 \text { si phase de l'expérience « aménités » (référence= phase } \\
\text { « conditions de production ») }\end{array}$ \\
\hline phregio & $\begin{array}{l}\text { Indicatrice : } 1 \text { si phase de l'expérience « région » (référence= phase } \\
\text { « conditions de production ») }\end{array}$ \\
\hline phgout & $\begin{array}{l}\text { Indicatrice : } 1 \text { si phase de l'expérience « goût » (référence= phase } \\
\text { « conditions de production ») }\end{array}$ \\
\hline $\begin{array}{l}\text { prix référence TB } \\
\text { prix référence TM }\end{array}$ & $\begin{array}{l}\text { Prix de référence déclaré pour la tome des Bauges } \\
\text { Prix de référence déclaré pour la tomme de montagne }\end{array}$ \\
\hline aménitésBaug & Note d'agrément des aménités des Bauges (moyenne des 7 photos) \\
\hline aménitésAPS & $\begin{array}{l}\text { Note d'agrément des aménités de l'Avant Pays Savoyard (moyenne des } 7 \\
\text { photos) }\end{array}$ \\
\hline $\begin{array}{l}\text { excurB } \\
\text { excurAPS }\end{array}$ & $\begin{array}{l}\text { Indicatrice : } 1 \text { si excursionniste dans les Bauges (référence=neutre) } \\
\text { Indicatrice : } 1 \text { si excursionniste dans l'Avant Pays Savoyard }\end{array}$ \\
\hline tourB & Indicatrice : 1 si touriste dans les Bauges (référence=neutre) \\
\hline sex & Indicatrice : 1 si homme \\
\hline hauteduc & Indicatrice : $1 \mathrm{si}$ « Bac+5 ou plus » (référence = bac à bac+4) \\
\hline faibleduc & Indicatrice : 1 si « au plus niveau lycée » (référence = bac à bac+4) \\
\hline ageinf & Indicatrice : 1 si âge $\leq 40$ ans (référence = de 41 à 60 ans) \\
\hline agesup & Indicatrice : 1 si âge> 60 ans (référence $=$ de 36 à 59 ans) \\
\hline revenuinf & $\begin{array}{l}\text { Indicatrice : } 1 \text { si revenu mensuel par unité de consommation }>2000 € \\
\text { (référence }=\text { de } 1000 \text { à } 2000 € / \text { mois) }\end{array}$ \\
\hline revenusup & $\begin{array}{l}\text { Indicatrice : } 1 \text { si revenu mensuel par unité de consommation <1000€ } \\
\text { (référence }=\text { de } 1000 \text { à } 2000 € / \text { mois) }\end{array}$ \\
\hline consorep & Indicatrice : 1 si consommation de fromage à tous les repas \\
\hline vsk & Indicatrice : 1 si recherche variété dans l'alimentation est forte \\
\hline sante & Indicatrice : 1 si niveau de préoccupation pour la santé alimentaire est forte \\
\hline enviro & $\begin{array}{l}\text { Indicatrice : } 1 \text { si niveau de préoccupation pour la préservation de } \\
\text { l'environnement est forte }\end{array}$ \\
\hline nouveau & $\begin{array}{l}\text { Indicatrice : } 1 \text { si niveau d'attrait pour de nouveaux produits alimentaires est } \\
\text { fort }\end{array}$ \\
\hline
\end{tabular}


Tableau 4. Influence de l'information, de l'appréciation du prix de référence, des aménités et des caractéristiques individuelles sur l'évaluation hédonique des fromages

\begin{tabular}{|c|c|c|c|c|c|c|c|}
\hline & ome des B & luges & & & nme de mor & ntagne & \\
\hline Variable & Estimation & Erreur star & ndard (1) & Variable & Estimation & Erreur stanc & dard (1) \\
\hline Intercept & 2.394 & 0.569 & 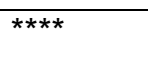 & Intercept & 2.665 & 0.656 & $\star * \star *$ \\
\hline phamen & 0.575 & 0.184 & $* * * *$ & phamen & 0.304 & 0.247 & \\
\hline phregio & 0.879 & 0.184 & $* * * *$ & phregio & 0.600 & 0.247 & $* * *$ \\
\hline phgout & 0.318 & 0.184 & $* *$ & phgout & 0.599 & 0.247 & $* * *$ \\
\hline prix référence TB & 0.060 & 0.011 & $* * * *$ & prix référence TM & 0.080 & 0.016 & $* * * *$ \\
\hline aménitésBaug & 0.609 & 0.090 & $* * * *$ & aménitésAPS & 0.258 & 0.100 & $* * *$ \\
\hline excurB & 0.309 & 0.165 & ** & excurAPS & 0.645 & 0.184 & $* * * *$ \\
\hline tourB & -0.588 & 0.180 & $* * * *$ & & & & \\
\hline homme & -0.004 & 0.140 & & homme & 0.297 & 0.192 & * \\
\hline hauteduc & 0.359 & 0.221 & * & hauteduc & -0.502 & 0.292 & $* *$ \\
\hline faibleduc & 0.541 & 0.178 & $* * * *$ & faibleduc & 0.147 & 0.237 & \\
\hline ageinf & 0.250 & 0.165 & * & ageinf & -0.209 & 0.219 & \\
\hline agesup & 0.085 & 0.194 & & agesup & -0.399 & 0.261 & * \\
\hline revenuinf & -0.811 & 0.246 & $* * * *$ & revenuinf & -1.056 & 0.329 & $* * * *$ \\
\hline revenusup & 0.022 & 0.162 & & revenusup & -0.039 & 0.220 & \\
\hline consorep & 0.275 & 0.149 & $* *$ & consorep & 0.190 & 0.202 & \\
\hline vsk & -0.384 & 0.134 & $* * * *$ & vsk & 0.063 & 0.182 & \\
\hline sante & 0.238 & 0.144 & ** & sante & 0.142 & 0.192 & \\
\hline enviro & 0.252 & 0.140 & ** & enviro & -0.123 & 0.183 & \\
\hline nouveau & -0.218 & 0.136 & * & nouveau & 0.266 & 0.182 & * \\
\hline $\mathrm{Nb}$ observations & 590 & & & $\mathrm{Nb}$ observations & 580 & & \\
\hline R2 & 0.266 & & & R2 & 0.1048 & & \\
\hline$F$ (p-value) & 10.86 & $(<.0001)$ & & $F(p$-value) & 3.65 & $(<.0001)$ & \\
\hline
\end{tabular}

(1) ****, ***, ** et * indiquent que les coefficients sont significativement différents de zéro avec un seuil de confiance respectivement de 1, 510 et 15\%.

L'estimation économétrique des CAP (cf. tableau 5) conduit à des résultats un peu différents. Certes, le prix de référence conserve son influence (il contribue à hauteur de $50 \%$ de la variance expliquée du CAP de la Tome des Bauges, $32 \%$ pour la tomme de montagne). L'impact de l'information fournie (aménités, région, goût) disparaît, excepté l'annonce de la région d'origine pour la Tome des Bauges. La note d'agrément des aménités paysagères n'a plus d'influence sur le CAP de la tomme de montagne; par contre, elle reste déterminante de la valeur économique de la Tome des Bauges. 
Tableau 5. Influence de l'information, de l'appréciation du prix de référence, des aménités et des caractéristiques individuelles sur le consentement à payer des fromages

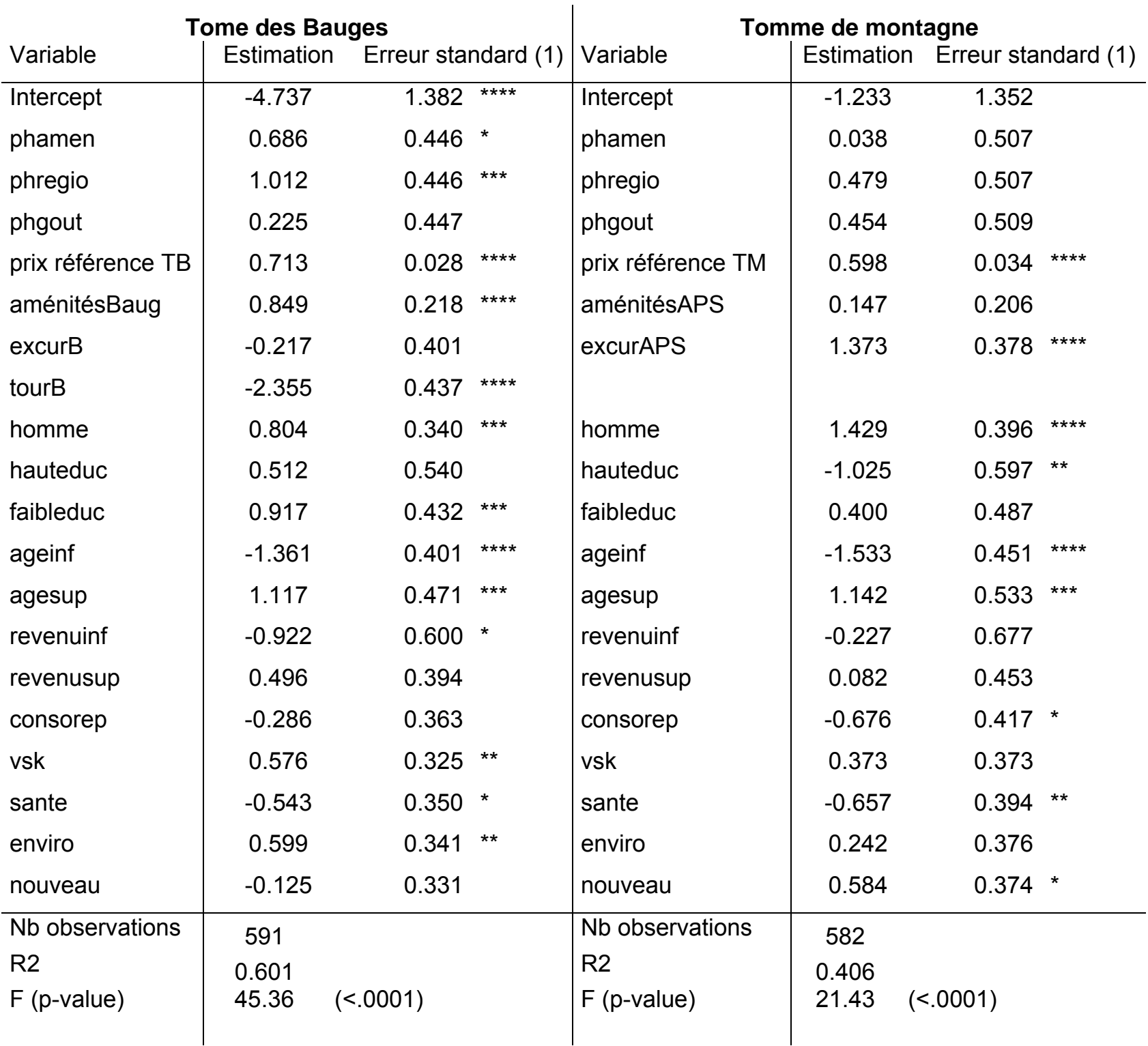

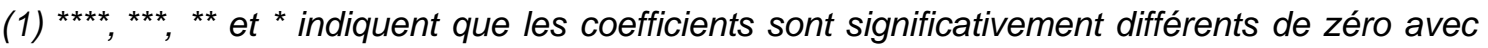
un seuil de confiance respectivement de 1, 510 et $15 \%$.

\subsection{La valorisation des aménités par les consommateurs}

En moyenne, les sujets ont davantage apprécié les aménités environnementales des Bauges que celles de l'Avant Pays Savoyard : les moyennes des mesures d'agrément des sept photos sont respectivement de 5,85 et de 5,09 et cette différence est significative au seuil de $1 \%$.

Les notes d'agrément de chacun des territoires expliquent les variations de "valeur affective " accordée à chacun des fromages : quand elles augmentent de 1 point, le score hédonique augmente de 0.3 pour la tomme de montagne et de 0.6 pour la tome des Bauges. Par contre, leurs impacts divergent sur leur « valeur économique » : il est nul sur le CAP de la tomme de montagne, alors qu'une variation de 1 de la note d'agrément pour les aménités des Bauges entraîne une variation de $0.85 € / \mathrm{kg}$ du CAP de la Tome des Bauges. 
Les participants ayant attribué une note supérieure à 6 (valeur médiane) aux aménités du massif des Bauges accordent une prime de 1,70 €/kg à la Tome des Bauges, toutes choses égales par ailleurs. Par contre, aucune prime n'est accordée à la tomme de montagne, dans le cas équivalent (estimations non présentées). En outre, la révélation de la zone de production permet de majorer de $1 €$ ce CAP. Cet effet peut s'expliquer par l'information fournie alors aux participants : " la tome est fabriquée dans le parc naturel régional du massif des Bauges, parc fondé sur la protection et la valorisation de son patrimoine naturel et culturel ». II est vraisemblable que l'on capture par ce biais la dimension humaine des aménités ${ }^{1}$, dimension qui s'ajouterait à celle de l'agrément des paysages.

\section{DISCUSSION}

Pourquoi l'attribut aménités prend-il une valeur dans le cas de la tome des Bauges et pas dans le cas de la tomme de montagne? Plusieurs éléments de réponse peuvent être apportés.

Tout d'abord les aménités du territoire des Bauges sont mieux évaluées que celles de l'Avant-Pays Savoyard et l'effet se propage aux évaluations hédoniques et économiques des produits.

Ensuite, on peut peut-être interpréter l'effet inversement proportionnel de la taille de la région sur la valeur du produit, effet mis en évidence par Stefani et al. (2006). Cet effet tiendrait au fait que plus le territoire est de petite taille, plus il est repérable et identifiable par les consommateurs. Bien que les deux territoires mobilisés dans notre expérience soient de taille similaire, leur identification n'est pas équivalente. Le massif des Bauges, dont les frontières naturelles sont saillantes, bénéficie d'une identité forte. A l'inverse, la délimitation floue de l'Avant Pays Savoyard ne permet pas une identification précise de ce territoire.

Enfin, l'effet de la congruence entre produit et territoire est à l'œuvre. Aurier et Fort (2005) montrent que l'effet de la congruence perçue entre le produit et le territoire est plus important que l'effet de l'origine seule. Ces auteurs constatent dans leur étude qu'il y a une congruence forte entre la tomme et la montagne. Or, dans le cas présent, les paysages présentés des Bauges évoquent davantage la montagne que ceux de l'Avant-Pays Savoyard.

La congruence n'a pas été mesurée ici, ce qui constitue une limite. II est cependant possible de supposer que la congruence entre la tomme de montagne et les paysages de son lieu de production est moyenne, car les paysages sont davantage agraires que montagnards. La vérification de cette hypothèse constituerait d'ailleurs un prolongement pertinent de cette recherche. Si le territoire n'est pas perçu par les consommateurs comme relevant d'un paysage de montagne (même s'il en fait partie du point de vue de la législation), il serait plus difficile de valoriser les produits sous ce nom là. Ceci renvoie à la perception de ce que sont les zones de montagne, et des distinctions entre définition des zones de production « montagne » et représentations des consommateurs.

Enfin, les différences entre les deux territoires sont à examiner de plus près, notamment à partir de la notion d'affordance (Caelen et Pellegrin, 2009 ; Gibson 1979). La mobilisation de cette notion qui correspond à une représentation de soi en action, met en évidence que l'appréciation du paysage est potentiellement plus évidente si le paysage correspond à une destination touristique ou un lieu à pratiquer. Ainsi, les images de territoire de montagne comme celles des Bauges, plus facilement associées à des destinations d'action, pourraient bénéficier de dimensions supplémentaires dans leur évaluation. Mais, c'est aussi par ce bais qu'on pourrait interpréter la prime accordée à chacun des fromages par les excursionnistes par rapport aux personnes ne pratiquant pas les territoires (cf. tableau 5).

\footnotetext{
${ }^{1}$ Huriot et Bourdeau-Lepage (2009) distinguent trois types d'aménités : les aménités environnementales, les aménités historiques et les aménités sociales, les deux dernières étant souvent interdépendantes.
} 
Ce travail ne constitue qu'une première approche des aménités et de leur valorisation potentielle via les produits alimentaires. II a permis de montrer que les aménités d'un territoire, même si elles participent de la valeur affective d'un produit, ne peuvent être valorisées économiquement que s'il y a congruence entre le territoire et le produit. Ces aménités seraient de deux ordres: naturelles, perçues via l'agrément des paysages notamment, et humaines, associées à l'évocation du nom du territoire. De ce fait, ce travail ouvre un champ de recherche très stimulant autour des liens entre territoire, produit, terroir et consommateur.

Plusieurs limites restreignent la portée de ce travail et mériteraient de conduire de nouvelles investigations. Les conclusions dressées ne sont valables que pour le produit étudié : le fromage. Elles ne peuvent être généralisées du fait de la spécificité-produit de l'effet du territoire mis en évidence par Van Ittertum et al (2003). En effet, ces auteurs ont montré que le transfert d'un produit à l'autre posait problème. La validité externe des résultats est donc réduite et ils appellent des réplications. L'appréhension théorique et la mesure des aménités environnementales perçues par les consommateurs doivent être affinées. Ici, elles ont été abordées de façon visuelle, et la convergence des modes de mesure est à étudier. Leur appréciation par les consommateurs n'a révélée qu'une seule dimension; toutefois, ce résultats peut être contingent aux photos sélectionnées et donc, les dimensions théoriques des aménités restent à valider, voire à compléter. L'évaluation du paysage pourrait être rapprochée de celles réalisées pour les destinations touristiques (Baloglu et McCleary, 1999; Echtner et Rictchie 2003 pour une revue): l'image organique d'un territoire, issue de l'information générale des individus y est distinguée de l'image induite par la promotion touristique des territoires (Gunn, 1988) et l'expéreince d'un territoire la transforme en image complexe. La question est ainsi posée du lien entre l'évaluation des aménités et les différentes images d'un territoire, et de l'effet de l'expérience.

Les déterminants individuels de l'appréciation des aménités sont également à creuser, notamment pour voir si des variables de personnalité ou de proximité avec le territoire modifient les évaluations. Le lien avec le sentiment régional, distinct de la familiarité, de l'appartenance ou de la pratique d'un territoire pourrait constituer aussi une voie de recherche intéressante. En effet, Dion et al. (2010) ont en effet montré que le sentiment régional exerce une influence importante sur la consommation des individus.

\section{BIBLIOGRAPHIE}

ABIVEN F., 2004, Caractérisation psycho-comportementale et repérage des segments de "goût» (consommateurs homogènes en terme de préférence gustative), Séminaire AFM / SYNTEC : Les métiers des Etudes : évolution des pratiques, Paris, 22 octobre.

ARTS-CHISS N \& GUILLON F., 2003, L'alimentation santé, un marché en voie de segmentation: une approche par les bénéfices produits et les risques perçu, Congrès sur les tendances du marketing, Paris.

AURIER P. et FORT F., 2005, Effets de la région d'origine, du produit, de la marque et de leurs congruences, sur l'évaluation des consommateurs : application aux produits agroalimentaires, Recherche et Applications en Marketing, 20, 4, 29-52.

BALOGLU S. AND MCCLEARY K., 1999, A Model of Destination Image Formation, Annals of Tourism Research, 26, 4, 688-697.

BeCKeR, G. M., DeGroot, M. H., \& MARSChAK, J., 1964, Measuring utility by a single response sequential method. Behavioral Science, 9(1), 226-232.

BOYLE, M.A., KIEL, K.A., 2001, A survey of house price hedonic studies of the impact of environmental externalities, Journal of Real Estate Literature, 9, pp.117-144.

CAELEN J. et Pellegrin A., 2009, Analyse cognitive des aménités environnementales, working paper, laboratoire LIG, Grenoble 
Cavailhès J., Brossard T., Foltête J.-C., Hilal M., Joly D., Tourneux F.-P., Tritz C., WAVRESKY P., 2009, GIS-Based Hedonic Pricing of Landscape, Environmental and Resource Economics, vol. 44, n 4, pp. 571-590.

CHEN Y., IRWIN E.G., JAYAPRAKASH C.L., 2009, Dynamic modeling of environmental amenitydriven migration with ecological feedbacks, Ecological Economics, 68(10), pp.2498-2510.

COHEN J.B. et ARENI C.S., 1991, Affect and consumer behavior, Handbook of consumer research, Prentice Hall.

CORDELlE S., LANGE C., ET SCHLICH P., 2004, On the consistency of liking scores :insights from a study including 917 consumers from 10 to 80 years old, Food Quality and preference, $15,831-841$.

CRELL M. et LOOMIS J., 1992, Recreation Value of Water to Wetlands in the San Joaquin Valley : Linked Multinomial Logit and Count Data Trip Frequency Model, Water Resources Research, vol. 28, n²10, pp. 2597-2606.

DELIZA, R., 1996, The effects of expectation on sensory perception and acceptance. Unpublished PhD thesis, University of Reading, UK.

DION D., REMY E. ET SITZ L., 2010, Le sentiment régional comme levier d'action marketing, Décisions Marketing, 58, à paraître.

DIREN Rhône-Alpes, 2007, Les 7 familles de paysages en Rhône-Alpes. Des paysages pluriels pour un territoire singulier, $36 \mathrm{p}$.

ECHTNER C ET RITCHIE J.R., 2003, The Meaning and Measurement of Destination Image, Journal of Tourisme Studies, 14, 1, 37-48.

GIBSON J.J. (1979) The ecological approach to visual perception. London: Lawrence Erlbaum

GuNN, C., 1988, Vacations Capes: Designing tourist regions. New-York: Van Nostrand Reinhold.

HANLey N., WRIGHT R. E. et KoOP G., 2002, Modelling Recreation Demand Using Choice Experiments : Climbing in Scotland, Environmental and Resource Economics, vol. 22, $\mathrm{n}^{\circ} 3$, pp. 449-466.

HURIOT \& BOURDEAU-LePAGE, 2009, Economie des villes contemporaines, éditions Economica, $380 \mathrm{p}$.

KOTCHEN M. J., 2005, Impure public goods and the comparative statics of environmentally friendly consumption.- Journal of Environmental Economics and Management, vol. 49, pp. 281-300.

LANGE C., 2000, Etude de l'effet des caractéristiques sensorielles, des attentes induites par l'information, et du prix sur l'acceptabilité et le comportement d'achat du consommateur, Thèse de doctorat en sciences de l'alimentation, Université de Bourgogne, $180 \mathrm{p}$.

LANCASTER K. J., 1966, A new approach to consumer society.- Journal of Political Economy vol. 74, pp. 132-157.

LEGALL-ELY M., 2009, Définition, mesure et déterminants du consentement à payer du consommateur : synthèse critique et voies de recherche, Recherches et Applications en Marketing, 24, 2, 91-113.

LEVIN, I. P., \& GAETH, G. J., 1988, Framing of attribute information before and after consuming the product. Journal of Consumer Research, 15, 374-378.

MOLLARD A., 2010, Les aménités environnementales: définitions et potentiels d'écodéveloppement territorial. Apports des cogniticiens et des écologues à une analyse économique de la valorisation des aménités, document de travail GAEL, $20 \mathrm{p}$. 
Mollard A., RambonilazA T., Vollet D., 2007, Environmental amenities and territorial anchorage in the recreational-housing rental market: A hedonic approach with French data, Land Use Policy, vol. 24, $\mathrm{n}^{\circ}$ 2, pp. 484-493.

MOREY E. R., 1981, The Demand for Site-Specific Recreational Activities : a Characteristics Approach , Journal of Environmental Economics and Management, vol. 8, n 4, pp. 345-371.

OCDE, 1994, La contribution des aménités au développement rural, publication OCDE, Paris, $99 \mathrm{p}$.

OCDE, 1996, Les aménités pour le développement rural - Exemples de politiques, publication OCDE, Paris, $130 \mathrm{p}$.

OCDE, 1999, Cultiver les aménités rurales : Une perspective de développement économique, publication OCDE, Paris, $122 \mathrm{p}$.

Poudyal N. C., Hodges D.G., 2008, The role of natural resource amenities in attracting retirees: Implications for economic growth policy, Ecological Economics, 68(1-2), pp. 240248.

SCHIFFerstein, H.N.J., Kole, A.P.W. and MoJeT, J., 1999, Asymmetry in the disconfirmation of expectations for natural yogurt. Appetite, 32, 307-329.

SIRIEIX L. (1999), Consommation alimentaire : problématiques, approches et voies de recherche, Recherche et Applications en Marketing, 14, 3, 41-58.

SKURAS D., DIMARA E., 2004, Regional image and the consumption of regionally denominated products.- Urban Studies, vol. 41, n 4, pp. 801-815.

SkuRas D., Dimara E., Petrou A., 2006, Rural tourism and visitors' expenditures for local food products.- Regional Studies, vol. 40, n 7, pp. 769-779

SpRENG, R. A.; MACKenZIE, S. B.; and Olshavsky,R. W., 1996, .A Reexamination of the

Determinants of Consumer Satisfaction,. Journal of Marketing, 60, 15-32.

StefanI G., Romano D., CAVICCHI A., 2006, Consumer expectations, liking and willingness to pay for specialty foods: Do sensory characteristics tell the whole story? Food Quality and Preference, vol. 17, $\mathrm{n}^{\circ}$ 1-2, pp. 53-62.

Steptoe A, Pollard T.M. \& Wardle J., 2005, Development of a measure of the motives underlying the selection of food : the food choice questionnaire, Appetite, 25, 267-284.

THORSNES P, 2002, The value of a suburban forest preserve: estimates from sales of vacant residential building lots, Land Economics, 78, pp.426-441.

Van ItTersum, K., Candel, M. J. J. M., Meulenberg, M. T. G., 2003, The influence of the image of a product's region of origin on product evaluation, Journal of Business Research, 56(3), pp. 215-226.

VAN TRIJP H.C.M. ET STEENKAMP J.B.E.M. , 1992, Consumer's variety seeking tendency with respect to foods : Measurement and managerial implications, European Review of Agricultural Economics, 19, 181-195.

VeRLEGH, P. W. J., SteENKAMP, J. E. M., 1999, A review and meta-analysis of country of origin research, Journal of Economic Psychology, 20, pp.521-546. 